\title{
Development of a FES System for Hemiplegic Rehabilitation Based on Patient Healthy Limb's EMG Signal
}

Zhan ZENG ${ }^{1, a}$, Chang-jun $\mathrm{LI}^{1}$, Chun-bao WANG ${ }^{2,3-6, b,{ }^{*}, \text { Quan-quan }}$ $\mathrm{LIU}^{2,3}$, Li-hong DUAN ${ }^{3,5}$, Tong-yang SUN ${ }^{2}$, Qing SHI ${ }^{4}$, Zhuo-hua LIN $^{4}$, Ya-jing SHEN ${ }^{5}$, Wan-feng SHANG ${ }^{7}$ and Zheng-zhi WU ${ }^{3, c,{ }^{*}}$

${ }^{1}$ School of Electronic Engineering and Automation, Guilin University of Electronic Technology, Guilin, 541004, China

${ }^{2}$ School of Mechanical and Automotive Engineering, South China University of Technology, Guangzhou, 510640, China

${ }^{3}$ Shenzhen Institute of Geriatrics, Shenzhen, China.

${ }^{4}$ Faculty of Science and Engineering, Waseda University, Japan.

${ }^{5}$ Mechanical and Biomedical Engineering, City University of Hongkong, China.

${ }^{6}$ Guangxi University of Science and Technology, China

${ }^{7}$ Faculty of Mechanical Engineering, Chinese University of Hongkong, China.

azhuzai815@126.com, bchunbaowang@163.com, 'szwzz001@163.com

${ }^{*}$ Corresponding authors

Keywords: FES, EMG, NRF24L01, Contralateral Control.

\begin{abstract}
This paper presents a functional electrical stimulation system to mimic the EMG characterization of patient healthy limb to exercise his/her affected limb. Firstly, the existing problems of functional electrical stimulation system are analyzed. Generally, the existing electrical stimulation systems suffer from fixed waveform, large volume and low portability, which make the effect of treatment difficult to improve. Secondly, the hardware platform of the proposed system is constructed, such as EMG acquisition module, FES module, to realize the acquisition of EMG signal and the generation of electrical stimulation pulse. The characterization of the healthy limb is extracted, and then it is used to exercise the affected limb via FES stimulus. Finally, the wireless communication is build, to realize the wireless communication between modules. The system has the advantage of small volume and high portable. The system can effectively collect EMG signals and generate FES pulses.
\end{abstract}

\section{Introduction}

Functional electrical stimulation (FES) plays an important role in rehabilitation of stroke patients as an important means of rehabilitation training for stroke patients[1]. Currently, the stimulus pulses used by FES are still specific synthetic pulses, such as single-phase, biphasic rectangular wave, triangular wave, sine wave, etc. These synthetic pulses do not accurately simulate the potential transfer of human muscle movement, affecting the treatment to further improve[2].

EMG signal is a more ideal source of stimulation to produce biological signals [3]. It is a good choice to use the normal EMG signal of the sound side as the stimulation source of the functional electrical stimulation ofaffectedside.

Because of the large volume and the cable connection, the existing electric stimulation system has the disadvantages of inconvenient, poor comfort and difficult 
carrying[4]. The use of wireless transmission instead of cable connection can be a good solution to these problems.NRF24L01 is a high-speed and easy-to-develop wireless communication module.NRF24L01 is suitable for small wireless transmission networks.

Therefore, this paper presents an electric stimulation system for hemiplegic patients using the contralateral EMG signal as a stimulus, combined with wireless transmission technology to improve the portability ofsystem.

\section{Design of System}

The system consists of EMG acquisition module, FES stimulation module, and master MCU. The data communication between modules is completed by NRF24L01. The system block is shown in Fig.1.

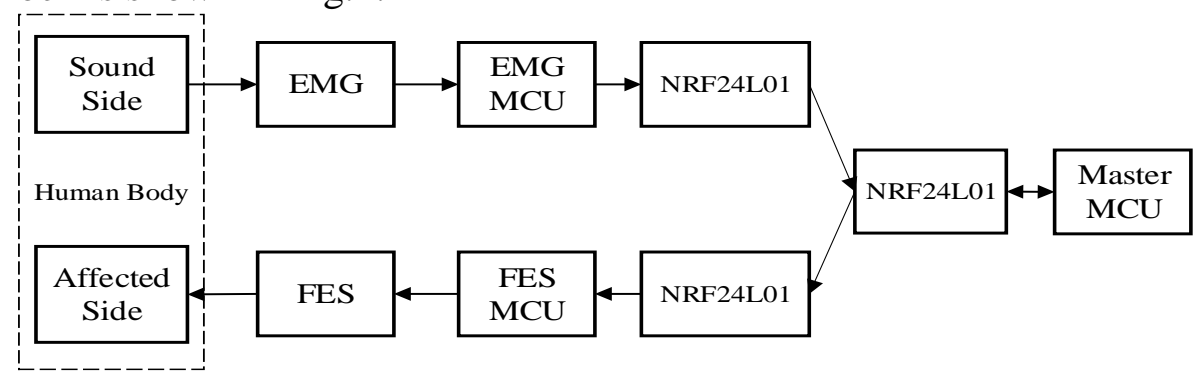

Figure 1. System block diagram

The EMG module is used for the processing of EMG signals. The EMG module includes front differential signal amplifier, high-pass filter, low-pass filter, main amplifier, power frequency trap and acquisition controller (EMG MCU). The FES stimulation module is used to generate electrical stimulation pulses. The FES stimulus pulse module consists of a boost circuit, a constant current control circuit, and a stimulus controller (FES MCU). Master MCU is used to analyze EMG signals and extract FES stimulus parameters. NRF24L01 is a wireless transmission module for wireless transmission of data.

\section{Design of System Hardware}

\section{EMG Module Hardware Design}

As a weak signal, the peak amplitude of surface EMG signal is generally only microvolt level. The useful energy of the signal is concentrated in the frequency range of 10 to $500 \mathrm{~Hz}$, mainly concentrated in the frequency range of $50 \sim 150 \mathrm{~Hz}$ [5]. The block diagram of EMG acquisition module is shown in Fig.2.

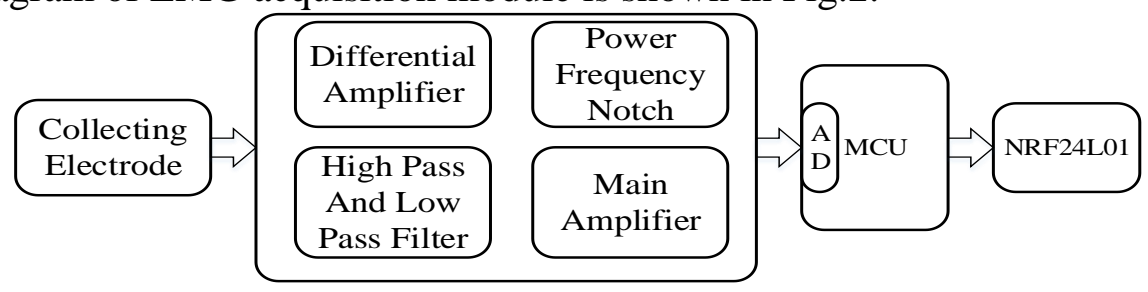

Figure 2. EMG acquisition system block diagram

Surface EMG picked up by the electrode containing a variety of noise, the EMG signal must be processed accordingly to remove the noise and to amplify the useful signal.In order to effectively suppress the interference of common-mode signals, the system adopts differential amplifier circuit.Amplifier circuit shows in Fig.3.The 
useful energy of the EMG signalisin the frequency range of 10 to $500 \mathrm{~Hz}$. For the effective extraction of EMG signals,band-passfilterneed to be designed.Theband-pass filter selects the Butterworth filter with the Sallen-Key structure.Filter circuit shows in Fig.5.The cutoff frequency formulashown as follow:

$$
\mathrm{f}=\frac{1}{2 \pi \sqrt{\mathrm{R}_{1} \mathrm{R}_{2} \mathrm{C}_{1} \mathrm{C}_{2}}} .
$$

Power frequency trap used to suppress the interference of the power frequency signal.Power frequency trapis a double-T band stop filter, shown in Fig.4.

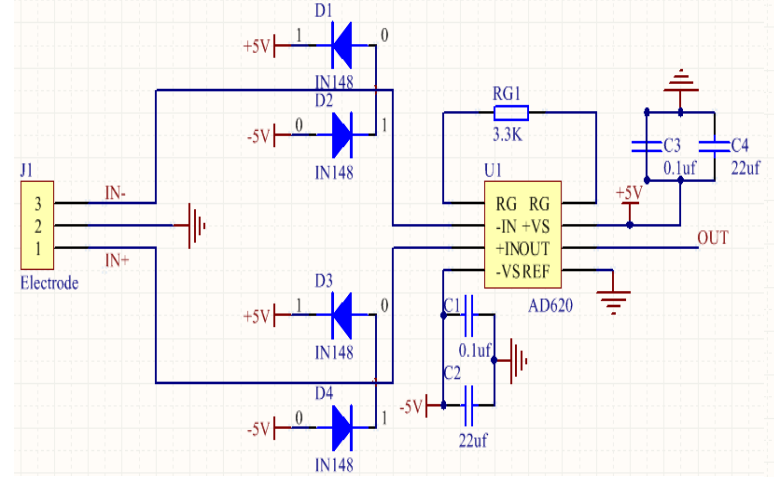

Figure 3. Amplifier circuit

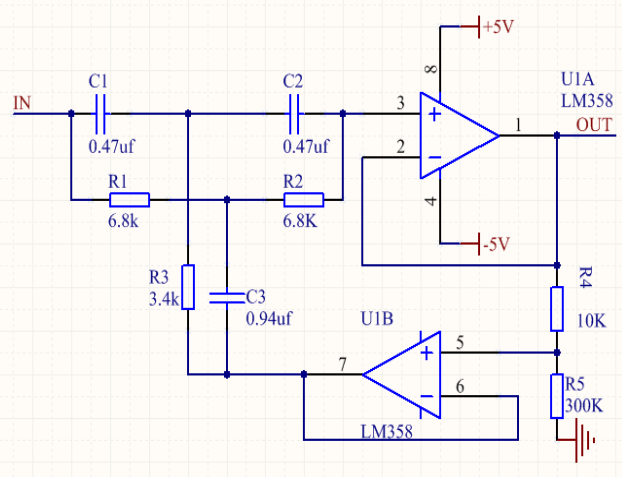

Figure4. Power frequency trap circuit

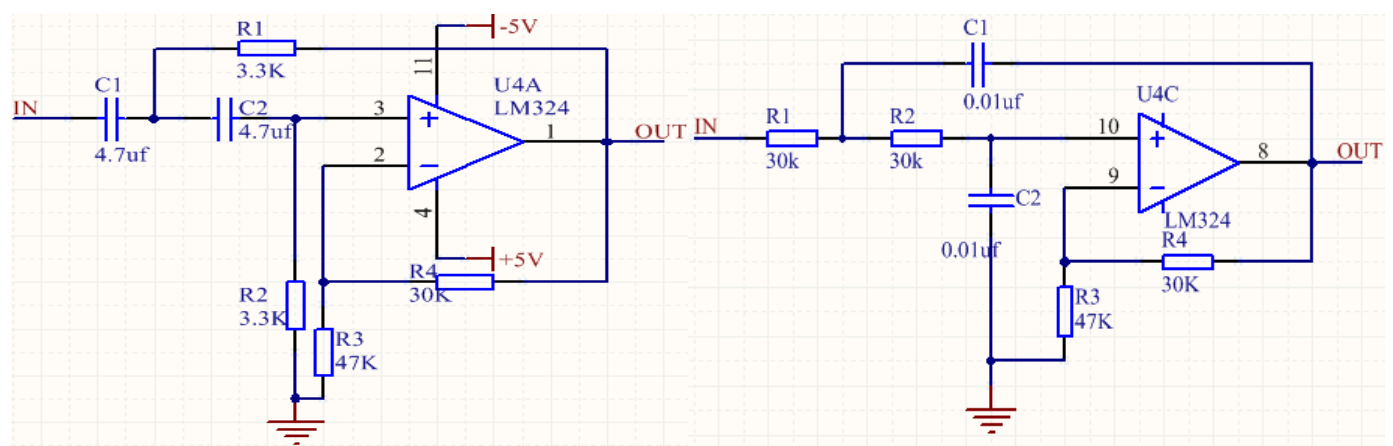

(a)High-pass filter(b)Low-pass filter

Figure5. Filter circuit

\section{FES Module Hardware Design}

The FES module is divided into the main controller, the boost circuit, the constant current control circuit, the wireless transmission module. Module block diagram shows in Fig.6.

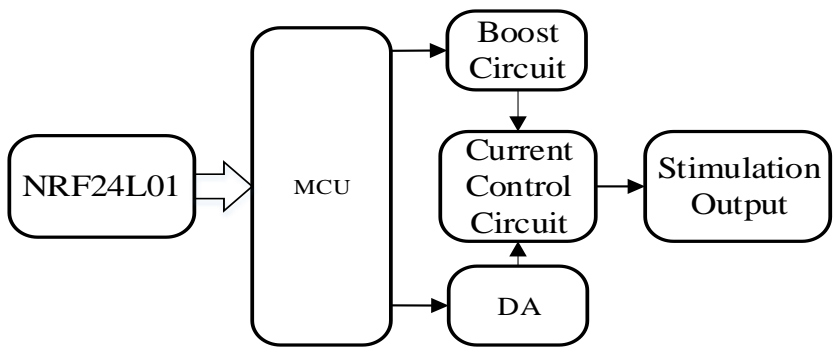

Figure6. Fes module block diagram

Because the impedance of skin, the stimulating voltage must be higher than a certain threshold to evoked action potential to cause motion reaction effectively. It is necessary to boost the low voltage DC power. The Boost circuit is shown in Fig. 7. 


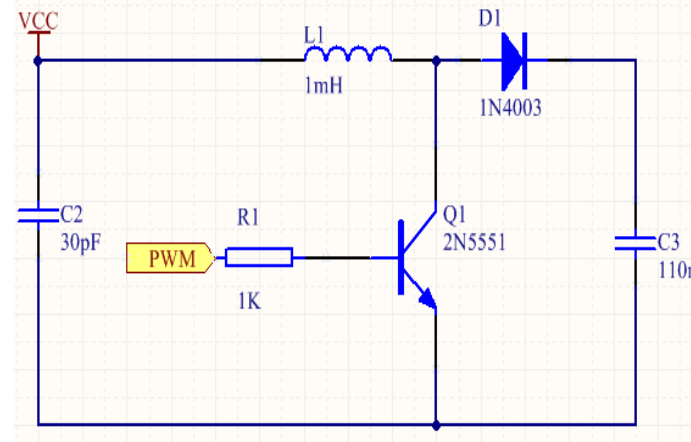

Figure7. FES boost circuit

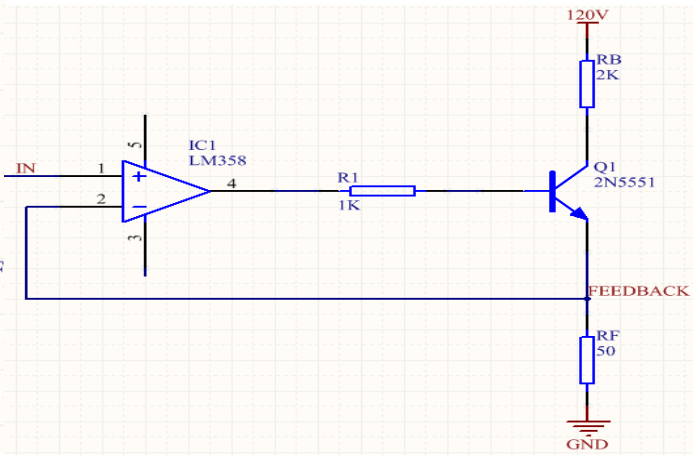

Figure8.Currentcontrol circuit

The control of the boost circuit can be realized by controlling the PWM pulse. Modifying the parameters of the PWM can adjust the level of the output voltage and realize the conversion from $4.5 \mathrm{~V}$ to $120 \mathrm{~V}$.

The resistance of the skin of the human body can vary depending on the position and the distance between the stimulating electrodes. To ensure that the current is maintained at a stable level when the resistance changes, it is necessary to design the current control circuit, shown in Fig. 8, body resistance is replaced by RB.

The relationbetween $\mathrm{I}_{\mathrm{RB}}$ and $\mathrm{U}_{\mathrm{IN}}$ can be expressed as the following formula:

$$
\mathrm{I}_{\mathrm{RB}}=\mathrm{I}_{\mathrm{RF}}=\frac{\mathrm{U}_{\mathrm{FEEDBACK}}}{\mathrm{RF}}=\frac{\mathrm{U}_{\mathrm{IN}}}{\mathrm{RF}} \text {. }
$$

The current IRF is determined by the voltage UIN and the resistor RF. By adjusting the input UIN can control the current IRF. Using DA conversion chip, FES MCU can stimulate the pulse according to the stimulus parameters.

\section{Control System and Wireless Transmission}

The master MCU is used to control the operation of the system, and to set parameters of EMG acquisition module and FES stimulation module through the NRF24L01 wireless transmission, Control System block diagram shown in Fig. 9. Themaster MCU receives the data of the EMG acquisition module, extracts the stimulation parameters of the FES electrical stimulation according to the characteristics of the EMG signal, and then transfers the stimulus parameters to the FES stimulus module. Flow chart ofsystem control shown in Fig.10.

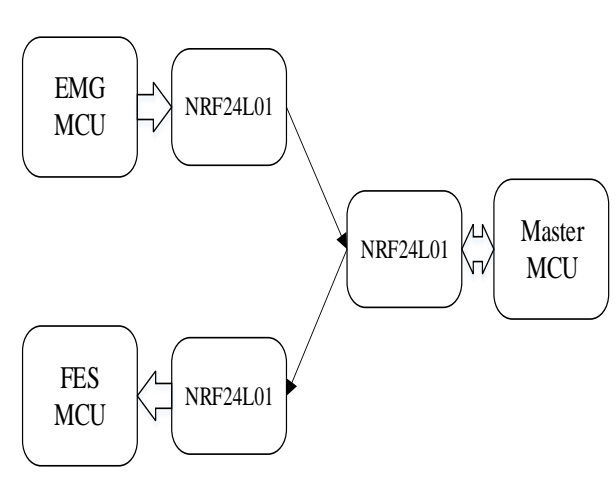

Figure9. Flow chartofsystem control

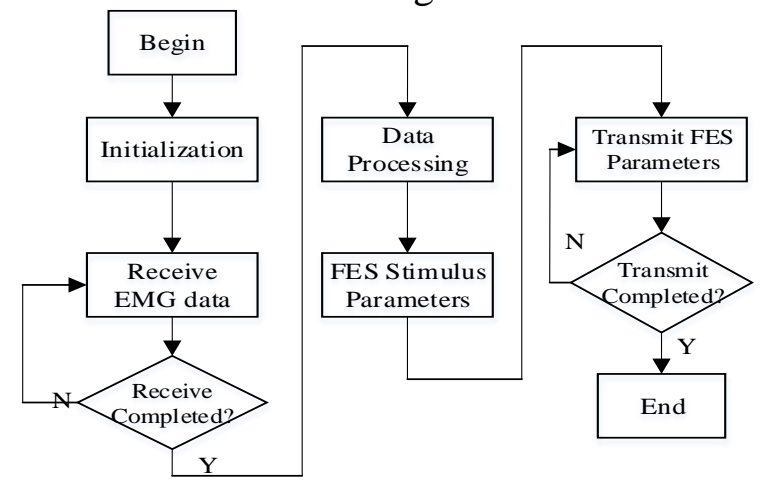

Figure10. Flow chartofsystem control 


\section{System Test Experiment}

\section{EMG Acquisition Module Test}

The EMG acquisition module can be tested by collecting arm EMG signals. The EMG signal of arm is tested respectively in in the state of motion and calm. And the oscilloscope used to show the EMG signal. The test results are shown in the Fig.11.

Test results show that the acquisition module can collect the body surface EMG signal, the muscle force process can also be reflected.

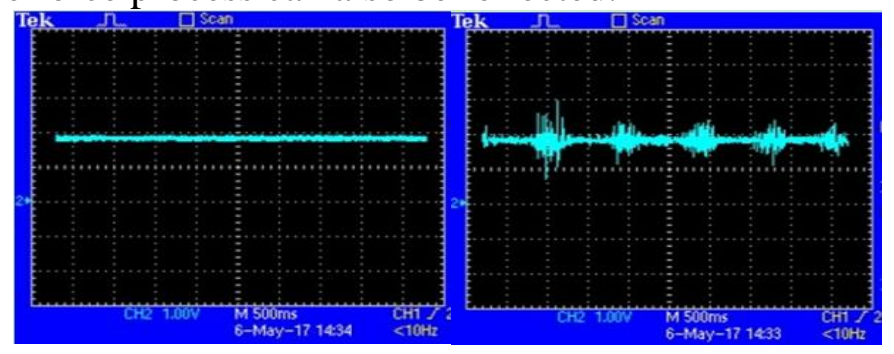

(a) Calm state(b) Movement state

Figure 11. Electromyography

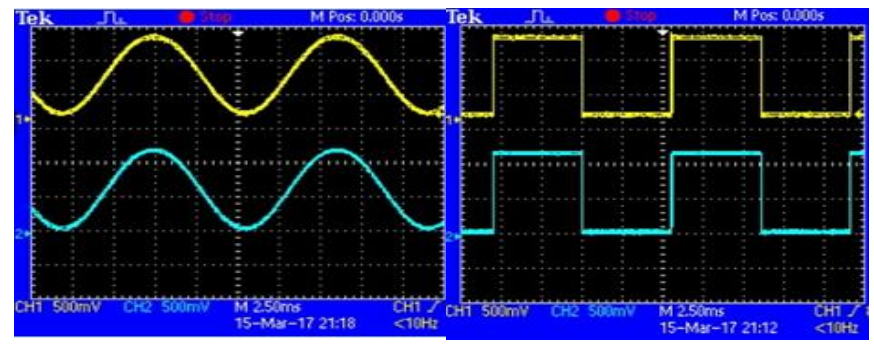

(a) Sine wave test(b) Rectangular wave test

Figure 12. Constant current control test

\section{Current Source Module Test}

The current through the resistor can be obtained by measuring the voltage across the resistor.The FES current control module is tested by usingsine waveand rectangular wave with frequency $90 \mathrm{~Hz}$, amplitude $1.2 \mathrm{~V}$. The test results are shown in the Fig.12. The yellow signal represents thevoltage of feedback resistance, the blue signal represents the input signal voltage.

FES output current is determined by the control signal, the output voltage changes with the control signal voltage amplitude changes. The current through the resistor is determined by the input signal.

\section{Conclusion}

This paper presents a FES electrical stimulation system using the contralateral EMG signal as the stimulus source, combined with wireless transmission technology. The system collects the EMG signals on the sound side of the hemiplegia patients, and uses the EMG signal as the pulse source to control the output of FES module on affected side. The EMG signal acquisition module is used to collect EMG signals, and the main control module is used to extract the FES stimulus parameters in the EMG signals, and the FES module generates stimulus pulses according to the stimulus parameters. NRF24L01 is used to realize wireless data communication between modules. The system can effectively improve the comfort and portability of functional electrical stimulation. It provides the hardware foundation for the further $\mathrm{t}$ research. 


\section{Acknowledgement}

The authors are thankful to the financial support from Science and Technology Foundation of Guangdong (No.2016A020220001, No.2014A020225004), Returned overseas business Foundation of Shenzhen (No.2016001), Technology Research Foundation of Basic Research Project of Shenzhen (No. JCYJ20160428110654601 and JCYJ20160428110354308), Research Foundation of Health and Family Planning Commission of Shenzhen Municipality (No.201601054), Medical Research Foundation of Guangdong (No.A2017250).

\section{References}

[1] Y. Yang, P. D. Li. Design of Real-time sEMG Feedback Type Electrical Stimulation Rehabilitation Training System. Progress in Biomedical Engineering,3(2015):142-145.(In Chinese)

[2] D.R.Merrill, M.Bikson,J.Jefferys, Electrical stimulationof excitable tissue, J Neuro Meth,141(2005):171-198.

[3] N. Lan,Z.X. Xiao, K.B. Nie.The principle, design andapplication of functional electrical stimulation. Chinese Journal of Rehabilitation Theory and Practice,4(1997):151-154.(In Chinese)

[4] Y.C. Chen,Y. Li,H. Li.Effect of Functional Electrical Stimulation on Hemiplegic Gait of Acute StrokePatients.Chinese Journal of Rehabilitation Theory and Practice, 2(2015):212-215. (In Chinese)

[5] Q. Kai.The Development of an Instrument for Contralaterally Controlled Functional ElectricStimulation.SoutheastUniversity,Nanjing,China,2016.(In Chinese) 